www.jmscr.igmpublication.org

Impact Factor 5.84

Index Copernicus Value: 83.27

ISSN (e)-2347-176x ISSN (p) 2455-0450

crossref DOI: _https://dx.doi.org/10.18535/jmscr/v5i3.131

Journal Of Medical Science And Clinical Research

\title{
Carba NP Test as a Rapid Diagnostic Tool for Carbapenemase Producers
}

\author{
Authors \\ Dalia H Abd El Hamid ${ }^{1 *}$, Sherin Ahmed EL-Masry ${ }^{2}$, \\ Amira Mohamed Mokhtar ${ }^{3}$, Eman Mohamed Kamel ${ }^{4}$
}

${ }^{1}$ MD Clinical Pathology, Lecturer of Clinical Pathology, Microbiology Unit, Faculty of Medicine.

Ain Shams University, Cairo, Egypt

${ }^{2}$ MD Clinical Pathology, Assistant Professor of Clinical Pathology, Microbiology Unit, Faculty of Medicine.

Ain Shams University, Cairo, Egypt

${ }^{3}$ MD Clinical Pathology, Professor of Clinical Pathology, Microbiology Unit, Faculty of Medicine.

Ain Shams University, Cairo, Egypt

${ }^{4}$ MD Clinical Pathology, Professor of Clinical Pathology, Microbiology Unit, Faculty of Medicine.

Ain Shams University, Cairo, Egypt

*Corresponding Author

Dalia Hosni Abd El Hamid

Email: dalia.mazen@med.asu.edu.eg,Phone number:00201223719886

Present Address: 11 Elgolf Street, $5^{\text {th }}$ Settlment, New Cairo, Cairo, Egypt

\begin{abstract}
The increasing resistance to carbapenems is a worldwide clinical concern. Resistance is mainly due to carbapenemases, which hydrolyse most $\beta$-lactams. Carbapenemases are found on diverse mobile genetic elements that may carry multiple resistance genes, leading to resistance to many antibiotic classes. Because of the complexity and diversity of carbapenem resistance mechanisms, particularly carbapenemase- mediated resistance, rapid and accurate detection is necessary to inform appropriate therapy. Carba NP and CarbAcineto NP tests are biochemical tests with relatively high sensitivities and specificities and faster turnaround times than culture based methods. The aim of this work is to detect the ability of Carba NP test to rapidly identify carbapenemase producing isolates. During this study, 100 carbapenem-resistant gramnegative isolates were subjected to Carba NP or CarbAcineto NP tests, subcultured on CHROMagar KPC media and tested by PCR for bla $a_{K P C}$, bla VIM $_{\text {, bla }}$ OXA, bla $a_{G E S}$, bla IMP. $_{94 / 100}(94 \%)$ isolates were positive for carbapenemase production by both Carba NP and CarbAcineto NP tests. 100/100 (100\%) were positive by CHRM agar KPC. 94/100 (94\%) isolates were positive for carbapenemase production by PCR. Carba NP test showed a sensitivity, specificity, PPV and NPV of 100\% compared to PCR in Enterobacteriaceae and Pseudomonas spp. CarbAcineto NP test showed a sensitivity, specificity, PPV and NPV of 100\% compared to PCR in Acinetobacterspp. CHRO Magar KPC test showed a sensitivity, specificity and PPV of 100\%, 0\%, and 94\% respectively compared to PCR. In conclusion, Carba NP and CarbAcineto NP tests are highly sensitive and specific, cheap, rapid tests for the detection of carbapenemase production.

Keywords: Carba NP test, KPC, CHROMagar KPC, CarbAcineto NP, Carbapenem.
\end{abstract}




\section{Introduction}

Carbapenems are commonly considered as lastresort antibiotics in the treatment of severe infections caused by multidrug-resistant gram negative bacteria. Over the past decade, the emergence of carbapenem-resistant isolates is very unnerving, since it contributes to the reduction of the current therapeutic pallet, therefore leaving few or, in some cases, no optimal therapeutic options ${ }^{(1)}$.

Although several mechanisms of carbapenem resistance have been reported, most of the mechanisms are related to the spread of carbapenemases belonging to Ambler class A (KPCs), class B (VIMs, IMPs, and NDM-1), and class D (OXA-48) $\beta$-lactamases ${ }^{(2)}$.

Bacterial isolates producing carbapenamasesare able to hydrolyze a broad spectrum of $\beta$-lactams including penicillins, cephalosporins, carbapenems and monobactam. They have the potential to spread rapidly in hospital environments to cause nosocomial infections with high mortality rates ${ }^{(3)}$, thus rapid identification of carbapenemase producing organisms is extremely important for timely detection, treatment and implementation of infection control measures to prevent their spread (4).

The detection of carbapenemases primarily on the basis of phenotypic testing may sometimes be difficult, since the MICs of carbapenem drugs may remain in the susceptibility range. Detection and identification of these carbapenem-resistant isolates may be improved significantly by the use of automated susceptibility testing systems ${ }^{(5)}$.

A series of non-molecular-based tests (e.g. modified Hodge test (MHT) and inhibition studies by EDTA and dipicolinic acid) have been proposed for detection of carbapenemase activity. None of the currently available tests has $100 \%$ specificity or $100 \%$ sensitivity ${ }^{(6)}$.

A variety of culture techniques have been proposed for screening of carbapenem resistant isolates including both in-house-prepared selective media in broth or solid agar form ${ }^{(7)}$ and commercially available agar plates either chromogenic or non- chromogenic media ${ }^{(8)}$. Chromogenic media containing a carbapenem are convenient tools for the screening and rapid detection of carbapenem-resistant GNB ${ }^{(9)}$.

Molecular techniques remain the reference standard for the identification and differentiation of carbapenemases. Most of them are based on PCR, and may be followed by sequencing for precise identification of a carbapenemase variants (e.g. VIM-type, KPC-type, NDM-type, and OXA48-type). They are either single PCR techniques or multiplex PCR techniques. The PCR technique performed on colonies can give results within 4-6 hours or less when real-time PCR technology is used, with excellent sensitivity and specificity ${ }^{(10)}$. Nevertheless, these molecular methods remain costly and, require special equipment and expertise in usage and result evaluation ${ }^{(1)}$.

Carba NP and CarbAcineto tests are biochemical tests with relatively high sensitivities and specificities. They were developed to detect carbapenemase producers in Enterobacteriaceae, Pseudomonas and A. Baumannii ${ }^{(11)}$. They have been used for attributing carbapenem resistance to the presence of a hydrolytic enzyme, instead of the MHT. This method was designed to identify the disruption of the $\beta$-lactam ring of the carbapenem substrate and uses a color variation, due to $\mathrm{pH}$ alteration, as an indicator. Data suggest that the Carba NP can detect carbapenemases belonging to all classes, regardless of MICs conferred, in a cost-effective, rapid and reproducible manner ${ }^{(12)}$.

The aim of this workis to detect the ability of Carba NP test to rapidly identify carbapenemase producing isolates, to detect its diagnostic performance in reference to molecular diagnosis and to compare it to chromogenic media as a rapid phenotypic detection method.

\section{Materials and Methods}

This study was conducted on 100 carbapenemresistant gram-negative isolates collected from different clinical samples submitted for routine culture and sensitivity in Central Microbiology 
Laboratory, Ain Shams University Hospitals. These isolates were identified by conventional microbiological techniques as colony morphology, Gram stain characteristics and biochemical reactions. Isolates were stored in tryptone soya broth with $15 \%$ glycerol at $-70^{\circ} \mathrm{C}$.

All isolates were sub cultured on Mueller Hinton agar, then subjected to:

1. Carba NP test (for Enterobacteriaceae and Pseudomonas spp.)which was performed according to the technique described by Nordman and his co-workers (2012)(12)in which Carba NP solution (A) was prepared by adding $2 \mathrm{~mL}$ of $0.5 \%$ phenol red solution to $16.6 \mathrm{~mL}$ sterile water with $180 \mu \mathrm{L}$ of $10 \mathrm{mmol} / \mathrm{L}$ zinc sulfate solution and adjusting the $\mathrm{pH}$ to $7.8+0.1$ with $0.1 \mathrm{~N}$ $\mathrm{NaOH}$ solution or $10 \% \mathrm{HCl}$ solution if $\mathrm{pH}$ is too high. The solution was then stored at 4 to $8^{\circ} \mathrm{C}$ in small vials and protected from prolonged light exposure for up to 2 weeks or not to exceed the expiry of individual components (the solution should be red, not to be used if turned to any color). This was used as a control solution and as a base for the test solution (B) which was prepared by adding $6 \mathrm{mg} / \mathrm{mL}$ imipenemcilastin powder to it and was stored at 4 to $8^{\circ} \mathrm{C}$ for up to 3 days.

The test procedure was carried in two micro centrifuge tubes ( $a$ and $b$ ) for each the tested isolate, quality control organism and uninoculated reagent control were labelled and $100 \mu \mathrm{l}$ of the TrisHCllysis buffer was added. For each isolate to be tested, $10 \mu 1$ loopful bacteria from an overnight Mueller Hinton agar plate was emulsified in both tubes " $A$ " and "B", vortexed for a minute and incubated at room temperature for 30 minutes. The bacterial suspension was centrifuged at $10,000 \times \mathrm{g}$ for 5 minutes. Uninoculated reagent control tubes should contain only bacterial lysis buffer. Organisms grown on selective media or plates containing antibiotics were not be used. In a micro well plate 2 wells were labelled as (a and b) for each isolate in which $100 \mu$ l of plain Carba NP (solution A) was added to well "a" and "b" and $100 \mu 1$ of Carba NP with imipenemcilastin (solution B) was added to well "b".30 $\mu 1$ of the supernatant, corresponding to the enzymatic bacterial suspension, was added to both well "a" and " $b$ " and the plate then was incubated at $35 \pm 2{ }^{\circ} \mathrm{C}$ for up to 2 hours.

\section{Interpretation}

- Both uninoculated control wells "a" and "b" must be red to red orange. If either was of any other color, the test was considered invalid.

- Inoculated well "a" must be red or red to orange. If it was of any other color, the test was considered invalid.

- Red or red to orange inoculated well "b" was considered negative for carbapenemase production.

- Light orange, dark yellow or yellow inoculated well "b" was considered positive for carbapenemase production (figure 1).

2. CarbA cineto NP test (for Acinetobacter spp.) In this test, the lysis buffer was replaced by a $5 \mathrm{M} \mathrm{NaCl}$ solution and the bacterial inoculum was doubled. It was done and interpreted as the Carba NP test (13).

3. Subculture on CHROMagar KPC media (CHROMagar, Paris, France) selective for carbapenemases producing organisms.

The isolates were streaked onto CHROMagar KPC using sterile bacteriological loops. Then the plates were incubated aerobically for 24 to $48 \mathrm{hrs}$ at $35 \pm 2^{\circ} \mathrm{C}$.

Carbapenem-resistant Enterobacteriaceae colonies appeared with different colors according to their specific enzymatic properties: Klebsiella spp., Enterobacter spp. And Citrobacter spp, as metallic blue, E.coli appears as red colonies, and 


\section{JMSCR Vol||05||Issue||03||Page 19133-19143||March}

Pseudomonas spp. as translucent cream colonies while Acinetobacter is cream, opaque colonies ${ }^{(14)}$

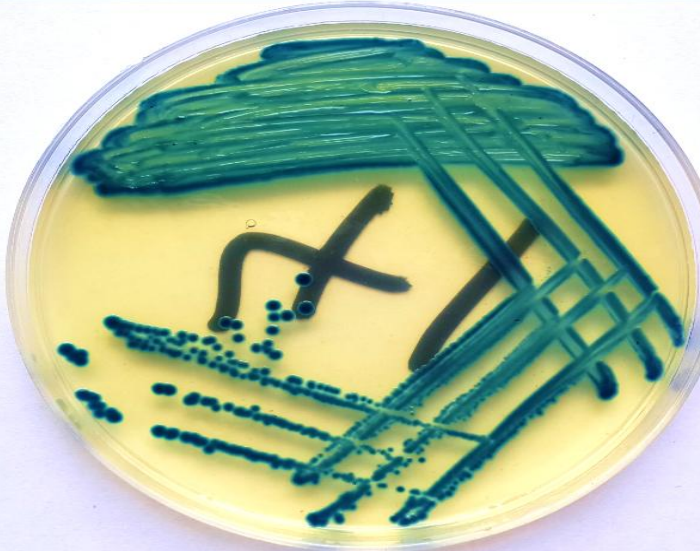

Figure (1a): Metallic blue colonies of Klebsiella, Citrobacteror Enterobacter.

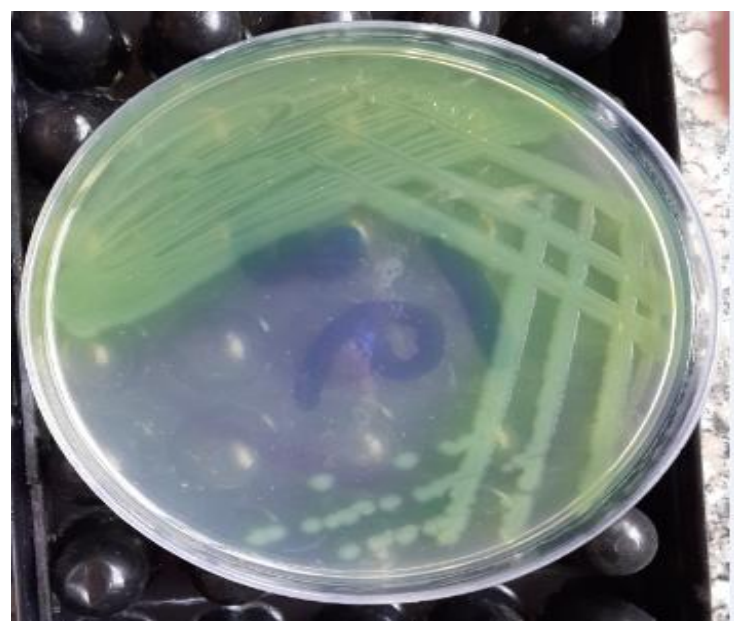

Figure (2c): Translucent cream colonies of Pseudomonas.
(Figure 2).

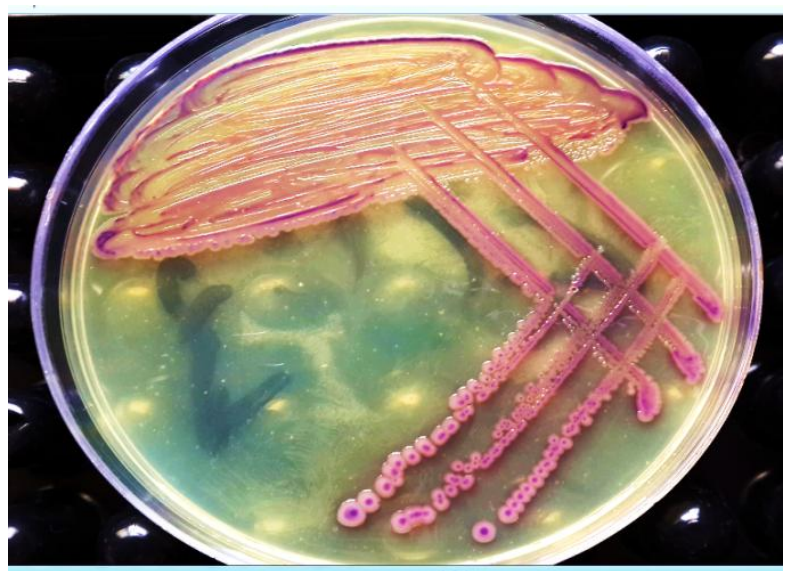

Figure (2b): Dark pink to red colonies of E.coli.

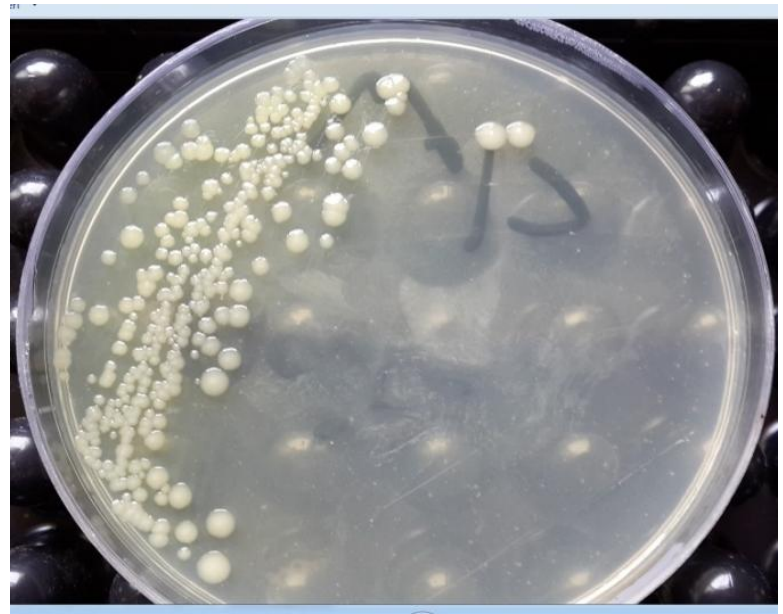

Figure (2d): Opaque cream colonies of Acinetobacter

Figure (2): Growth on CHROMagar KPC

4. Polymerase chain reaction for the identification of the most common types of serine carbapene-mases and MBLs according to Monterio and his collegues $(2012)^{(15)}$. Genes identified are KPC, IMP, OXA-48, VIM and GES.

Polymerase chain reaction was done using Qiagen Roter gene 5plex (Germany). Bacterial DNA was extracted using DNA extraction kit QIA amp

\begin{tabular}{|l|}
\hline Forward Primers \\
\hline bla ${ }_{\text {KPC }}$ type 5`TCGCTAAACTCGAACAGG \\
\hline blaGEStype 5`CTATTACTGGCAGGGATCG \\
\hline blaOXA-48 type5`TGTTTTTGGTGGCATCGAT \\
\hline blaIMP type 5`GAGTGGCTTAATTCTCRATC \\
\hline blaVIM type 5`GTTTGGTCGCATATCGCAAC \\
\hline
\end{tabular}

The amplification was done as described by (Monterio et al., 2012$)^{(15)}$. The thermal cycler was
DNAMini Kit (Qiagen, USA). The reaction mixture included Quanti Tect SYBR ${ }^{\circledR}$ Green PCR Kits (Qiagen, USA), Oligonucleotide primers (Qiagen, USA),Template DNA and Nuclease-free waterin a final volume of $25 \mu \mathrm{L}(15)$. K. pneumonia ATCC 1705 was used as a positive control and $K$. pneumonia ATCC 1706 as a negative control (supplied by NAMRU 3)

Reverse Primer
BlaKPCtype 5`TTACTGCCCGTTGACGCCCAATCC
blaGES type 5`CCTCTCAATGGTGTGGGT
blaOXA-48 type 5`GTAAMRATGCTTGGTTCGC
blaIMP type 5`AACTAYCCAATAYRTAAC
blaVIM type 5`AATGCGCAGCACCAGGATAG

programmed with initial denaturation step at $95^{\circ} \mathrm{C}$ for $5 \mathrm{~min}$ and 45 cycles for amplifications 
consisting of denaturation at $95^{\circ} \mathrm{C}$ for $20 \mathrm{sec}$, annealing at $55^{\circ} \mathrm{C}$ for $45 \mathrm{sec}$, and extension for 30 seconds. A positive result is considered when a cycle threshold $(\mathrm{Ct})$ of 40 or less and a negative result for a $\mathrm{Ct}$ of more than 40 . The amplification program was followed immediately by a melting program (to detect primer dimer) consisting of 1 minute at $95^{\circ} \mathrm{C}, 30 \mathrm{sec}$ at $55^{\circ} \mathrm{C}$ then again to $95^{\circ} \mathrm{C}$ for $30 \mathrm{sec}$.

\section{Data Management and Analysis}

Non-numerical data were expressed as numbers (\%). Chi-square was used to compare between two variables in qualitative data. Fisher exact test was used to examine the relationship between two qualitative variables when the expected count is less than 5 in more than $20 \%$ of cells in which Pvalue less than 0.05 is considered non-significant (NS), $\mathrm{P}<0.05$ is Significant ( $\mathrm{S}$ ), and $\mathrm{P}<0.01$ is Highly significant (HS). All the analyses were performed with commercially available software (SPSS 15.0.1 for windows; SPSS Inc, Chicago, IL, USA 2001).

The diagnostic performance of the Carba NP test, CarbAcineto NP test and CHROMagar KPC was determined according todiagnostic sensitivity, diagnostic specificity, predictive value for a positive test (PPV), predictive value for a negative test (NPV).

\section{Results}

The studied isolates included 47 Acinetobacter spp., 34 Enterobacteriaceae spp. and 19 Pseudomonas spp.

Both Carba NP and PCR were positive in 94 isolates out of 100 studied isolates while CHROMagar KPC was positive in all studied isolates.

\section{Polymerase chain reaction}

Any isolate was positive for one or more of the 5 tested genes considered positive for PCR in which total positive was $94 \%$ while $6 \%$ were negative for all tested genes. (data of distribution of different genes among different isolates is not shown)

\section{Carba NP}

Both Carba NP test and PCR could detect carbapenemase production in 30/30 Enterobacteriaceae isolates (100\%) and were negative in 4/4(100\%). Therefore, Carba NP test has sensitivity, specificity, PPV and NPV of $100 \%$ compared to PCR (table1,2)

There was a highly significant statistical association between Carba NP test and PCR in Enterobacteriaceae $(\mathrm{P}<0.001)$ as thirty $(100 \%)$ isolates were positive and four $(100 \%)$ isolates were negative by both tests.

When evaluating the use of Carba NP test in the detection of carbapenemase production in Pseudomonas spp., we found that among the nineteen tested isolates 18/18(100\%) were positive by both Carba NP and PCR and only one isolate was negative $1 / 1(100 \%)$ by both tests. So, the sensitivity, specificity, PPV and NPV of Carba NP test were found to be $(100 \%, 100 \%$, $100 \%$, and $100 \%$ ) respectively (table 1,2$)$.

A significant statistical association $(\mathrm{P}=0.05)$ between Carba NP test and PCR in Pseudomonas was found as eighteen (100\%) isolates were positive and one $(100 \%)$ isolate was negative by both tests.

\section{CarbAcineto NP}

When coming to detect carbapenemase production in Acinetobacter spp., CarbAcineto NP test was used because Carba NP test failed to detect OXA and GES carbapenemases, which are the most frequently identified carbapenemases among Acinetobacter spp. according to recommendations by(13)

Among 47 Acinetobacter isolates tested for carbapenemase production by CarbAcineto NP test, 46 were positive by both CarbAcineto and PCR $(100 \%)$ and one isolate was negative by both tests. So, the sensitivity, specificity, PPV and NPV of $(100 \%, 100 \%, 100 \%$, and $100 \%)$ respectively (table1,2)

There was a highly significant statistical association $(\mathrm{P}<0.001)$ between CarbAcineto NP test and PCR in Acinetobacter as forty-six (100\%) 
isolates were positive and one (100\%) isolate was negative by both tests.

\section{CHROMagar KPC}

All the one hundred carbapenem resistant isolates grew on CHROMagar KPC media as shown in table (3).
As regards CHROMagar KPC, Both CHROMagar KPC and PCR were positive in $94 \%$ of cases. While CHROMagar KPC was positive in $6 \%$ of cases, which were negative by PCR(table3).It gave a sensitivity, specificity and positive predictive value of $(100 \%, 0 \%$ and $94 \%)$ respectively.

Table (1): Results of Carba NP and CarbAcineto NP tests in relation toPCR in Enterobacteriaceae, Pseudomonas and Acinetobacter spp.

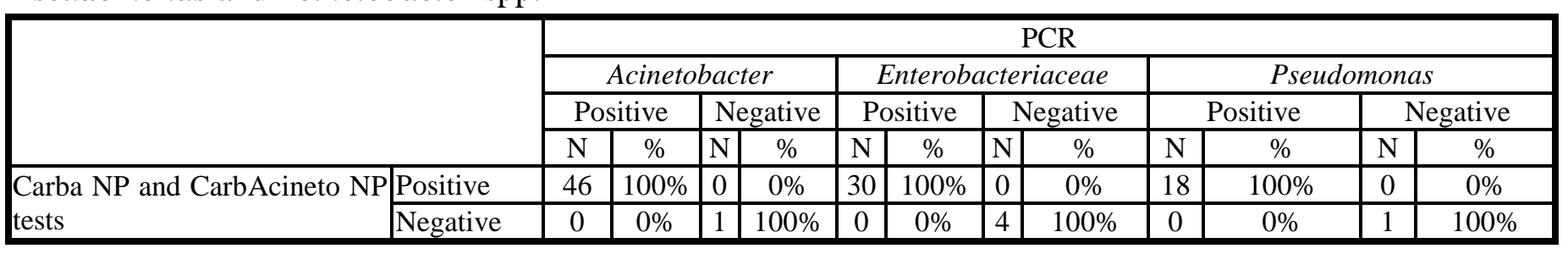

Table (2): Diagnostic performance of Carba NP test,CarbAcineto NP test and CHROMagar KPC compared to PCR

\begin{tabular}{|l|c|c|c|c|}
\hline & Sensitivity & Specificity & PPV NPV \\
\hline Carba NP test & $100 \%$ & $100 \%$ & $100 \%$ & $100 \%$ \\
\hline CarbAcineto NP test & $100 \%$ & $100 \%$ & $100 \%$ & $100 \%$ \\
\hline CHROMagar KPC & $100 \%$ & $0 \%$ & $94 \%$ & - \\
\hline
\end{tabular}

Table (3): Results of CHROMagar KPC in relation to PCR results

\begin{tabular}{|c|c|c|c|c|c|c|c|}
\hline & & \multicolumn{4}{|c|}{ PCR } & \multirow{2}{*}{\multicolumn{2}{|c|}{ Total }} \\
\hline & & \multicolumn{2}{|c|}{ Positive } & \multicolumn{2}{|c|}{ Negative } & & \\
\hline & & No & $\%$ & No & $\%$ & No & $\%$ \\
\hline \multirow{2}{*}{ CHROMagar KPC } & Positive & 94 & $100 \%$ & 6 & $100 \%$ & 100 & $100 \%$ \\
\hline & Negative & 0 & $0 \%$ & 0 & $0 \%$ & 0 & $0 \%$ \\
\hline \multicolumn{2}{|l|}{ Total number } & 94 & $100 \%$ & 6 & $100 \%$ & $100 \%$ & $100 \%$ \\
\hline
\end{tabular}

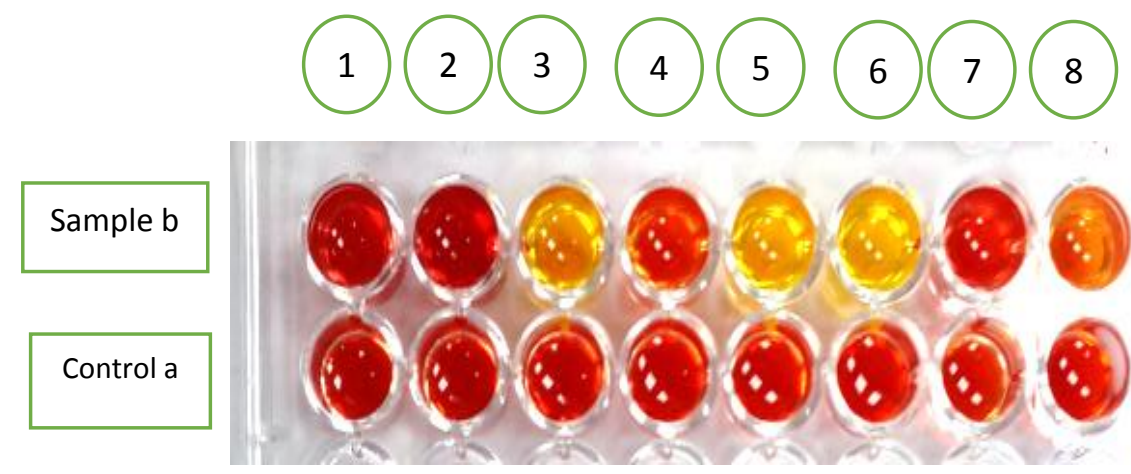

Figure (2):Carba NP test. Well 1: un-inoculated control, 3, 5, 6, 8 show positive results. Wells 2, 4, 7 show negative results.

\section{Discussion}

The results of Carba NP test in Entrobacteriaceae in reference to PCR came in agreement with a study by Nordmann et al., (2012) in
France which reported strongly consistent results of the Carba NP test and the real-time PCR, as the sensitivity and specificity of the Carba NP test compared to the real-time PCR were 
(162/162) $100 \%$ and (47/47) $100 \%$ respectively (12)

Also, in another study in USA by Vasoo et al., (2013) found that the sensitivity of the Carba NP test in detecting carbapenemase production in Enterobacteriaceae compared to PCR was $(115 / 115) 100 \%$ and the specificity was $(29 / 29)$ $100 \%{ }^{(16)}$.

On the other hand, other publications showed different results as a study conducted in Canada by Tijet et al.,(2013) on Enterobacteriaceae, who found that both the specificity and positive predictive value of the Carba NP test were (84/84) $100 \%$ in comparison to PCR. While its sensitivity and negative predictive value were (105/132) $72.5 \%$ and $69.2 \%$, respectively, and increased to $80 \%$ and $77.3 \%$, respectively, when a more concentrated bacterial extract, by increasing the amount of bacteria used to three to four calibrated $10 \mu 1$ loopfuls instead of one, was used $^{(17)}$.

Also the results of the current study wereslightly different from those of Yusuf and his colleagues (2014)in Belgium as they reported thatthe sensitivity, specificity, PPV and NPV of the Carba NP test using Enterobacteriaceae isolates were (41/45) $91.1 \%$, (47/47) 100\%, 100\%, $92.2 \%$ respectively compared to PCR ${ }^{(18)}$.

Our results showed slight difference from those of Knox and his colleagues (2014) in Australia who evaluated the performance of Carba NP test in detecting carbapenemase production by Enterobacteriaceae in comparison to PCR and found that the sensitivity and specificity of the Carba NP test were (40/46) $87 \%$ and (59/59) $100 \%$ respectively ${ }^{(19)}$.

The variation in sensitivity of the Carba NP test may be attributed to many factors such as the difference in the prevalence rate of carbapenemases with low hydrolysis activity to imipenem in each area (particularly OXA and some IMP enzymes) and low carbapenemase gene expression in some isolates. Also, mucoid colonies (difficulties in protein extraction), species-specific traits (due to the unknown impact of different genetic backgrounds), types of agar media (MacConkey agar media is not recommended) or imipenem powder used as therapeutic intravenous (I.V.) imipenemcilastatin versus reference standard imipenem powder which may play a role $(17 ; 20 ; 21 ; 22)$.

Despite the fact that we had nineteen Pseudomonas isolates with only one negative isolate by Carba NP test, it was found to be very consistent and acceptable when compared to other studies as in a study conducted by Yusuf and his coworkers (2014) in Belgium who had also a total of nineteen isolates of Pseudomonas. They found that the sensitivity (6/6), the specificity (13/13), PPV and NPV of the Carba NP test were $100 \%$ respectively compared to PCR ${ }^{(18)}$.

On the other hand, slightly different results were seen in a study done by Dortet et al., (2012) in France which showed that the sensitivity and specificity of the Carba NP test in detecting carbapenemase production in Pseudomonas spp. $\begin{array}{lllll}\text { were }(34 / 36) & 94.4 \% & \text { and (72/72) } & 100 \%\end{array}$ respectively, compared to PCR. The decreased sensitivity was due to failure of the Carba NP test to detect two GES producing isolates(23).

In addition, our results were a bit different from those of Huang and his coworkers (2014) who evaluated the ability of Carba NP test to detect carbapenemase production in Pseudomonas spp. in a study in Belgium. They found that the sensitivity, specificity, PPV and NPV of the Carba NP test were (20/22) 91\%, (13/13) 100\%, $100 \%$, and $96 \%$ respectively compared to PCR. The decreased sensitivity was due to failure of the Carba NP test to detect two OXA producing isolates(24).

In Belgium,another study done by Heinrichs and his colleagues (2015). They reported that the sensitivity, specificity, PPV and NPV of the Carba NP test in detecting carbapenemaseproducing Pseudomonas were (66/75) $88 \%$ and $(112 / 113) 99 \%, 98 \%$ and $95 \%$ respectively, compared to PCR. The decreased sensitivity was due to failure of the Carba NP test to detect nine 
(two IMP-13, one GES-18, four OXA-198 and two VIM-2) carbapenemase producing isolates (25)

The variation in sensitivity of Carba NP test in detecting carbapenemase production by Pseudomodnas spp. is mostly due to the production of weak carbapenemases such as OXA-48, GES and IMP $(23 ; 24 ; 25)$.

Dortet et al., (2014) found that the sensitivity and specificity of the CarbAcineto NP test in detecting carbapenemase production by Acinetobacter spp. were (143/151) $94.7 \%$ and (69/69) $100 \%$ respectively, compared to PCR in a study done in France. The lower sensitivity reported is due to failure of the test to detect eight GES producing isolates ${ }^{(13)}$.

In our study, we could not study the impact of each individual gene on the sensitivity and the specificity of the Carba NP and the CarbAcineto $\mathrm{NP}$ tests as mostof the tested isolates were carrying more than one gene.

The results of CHROMagar KPC shows that it is a good media for detection of carbapenem resistance but cannot identify whether it is due to carbapenemase production or due to other mechanisms. Therefore, the specificity for detection carbapenemase production was $0 \%$. However, further identification of the cause of resistance might be needed for epidemiological purposes in special situations. Yet, it can be recommended for rapid screening for carbapenem resistance in clinical settings.

The false positive results by CHROMagar KPC may be due to the presence of other mechanisms of carbapenem resistance, such as modifications of the outer membrane permeability or upregulation of efflux systems associated with hyperproduction of AmpC $\beta$-lactamases and cephalosporinases (ESBLs) ${ }^{(9)}$.

However, our results came in disagreement with those of Adler et al. (2011) in Israel who reported that the sensitivity of detection of carbapenemase producers by CHROMagar KPC was $84.9 \%$ and its specificity was $88.7 \%$, compared to PCR. The sensitivity of CHROMagar KPC was low because it could identify only 28/33 carbapenem resistant isolates ${ }^{(26)}$.

In addition, our results were different from those reported by Samra et al., (2008) in Isrealas theyfound that CHROMagar KPC media was $100 \%$ sensitive and $98.4 \%$ specific in detecting carbapenem resistance in Enterobacteriaceae compared to PCR. The decreased specificity of CHROMagar KPC media was due to the growth of carbapenem resistant isolates that were negative by PCR for Carbapenemase production (27)

Another study conducted in Israel by Gilad and his coworkers (2011) evaluated CHROMagar KPC for 53 carbapenem-non susceptible strains. They found that KPC-producing isolatesgrew efficiently ( $100 \%$ sensitivity). However poor or no growth or atypical colony color were evident with carbapenem resistant KPC nonproducing isolates( $72.7 \%$ sensitivity). Therefore, they concluded that CHROMagar KPC is applicable for processing carbapenem-resistant Enterobacteriaceae surveillance specimens, but its performance might possibly be suboptimal in the presence of KPC-negative carbapenemresistant strains ${ }^{(28)}$. However, in our study KPC gene was detected in only $17 \%$ of the tested isolates but CHROM agar showed growth in all tested isolates which disagrees with their conclusion.

\section{Conclusion}

Carba NP and CarbAcineto NP tests are inexpensive, rapid ( $2 \mathrm{hrs}$ ), reproducible and highly sensitive and specific methods for detection of carbapenemase production from bacterial colonies grown on culture media when compared to molecular techniques as a reference method, PCR is also rapid and highly sensitive method in detection of Carbapenemase production, however it is more expensive and needs trained personnel with special equipment.

Carba NP and CarbAcineto NP testscan perfectly differentiate carbapenemase producers from strains that are carbapenem resistant due to non 
carbapenemase-mediated mechanisms. They eliminate the need for using other techniques, which are time-consuming and less sensitive or specific as CHROMagar KPC, which remains an easy and cost effective screening method for the detection of carbapenem resistance in clinical settings, yet it is less specific for carbapenemase production than Carba NP test and cannot differentiate carbapenemase producers from strains that are carbapenem resistant due to non carbapenemase-mediated mechanisms.

\section{References}

1. Voulgari E, Poulou A, Koumaki V, et al., (2013): Carbapenemase-producing Enterobacteriaceae: now that the storm is finally here, how will timely detection help us fight back? Future Microbiology; 8(1): 2739.

2. Nass T, Cuzon G, Bogaerts P, et al., (2011): Evaluation of a DNA Microarray (Check-MDR CT102) for Rapid Detection of TEM, SHV, and CTX-M ExtendedSpectrum $\beta$-Lactamases and of KPC, $O X A$ 48, VIM, IMP, and NDM1Carbapenemases. Journal of Clinical Microbiology; 49(4): 1608-1613.

3. Wang L, Gu H and Lu X (2012): Rapid low-cost detection of Klebsiellapneumoniae carbapenemase genes by internally controlled real-time PCR. Journal of Microbiological Methods; 91: 361-363.

4. Pragasam AK, Sahni RD, Anandan S, et al., (2016): A Pilot Study on Carbapenemase Detection: Do We See the Same Level of Agreement as with the CLSI Observations. Journal of Clinical and Diagnostic Research; 10(7): DC09DC13.

5. Nordmann P, Poirel L, Toleman MA, et al., (2011): Does broad-spectrum betalactam resistance due to NDM-1 herald the end of the antibiotic era for treatment of infections caused by Gram-negative bacteria? Journal of Antimicrobial Chemotherapy; 66(4): 689-92.

6. Nordmann P, Gniadkowski M, Giske CG, et al. (2012): Identification and screening of Carbapenemase-producing Enterobacteriaceae. Clinical Microbiology and Infection; 18(5): 432-438.

7. Calfee D and Jenkins SG (2008): Use of active surveillance cultures to detect asymptomatic colonization with carbapenem-resistant Klebsiellapneumoniae in intensive care unit patients. Infection Control Hospital Epidemiology;29(10): 966-968.

8. Vrioni G, Daniil I,Voulgari E, et al. (2012): Comparative evaluation of a prototype chromogenic medium (ChromID CARBA) for detecting carbapenemase-producing Enterobacteriaceaein surveillance rectal swabs. Journal of Clinical Microbiology; 50(6): 1841-1846.

9. Simner PJ, Gilmour MW, DeGagne P, et al. (2015): Evaluation of Five Chromogenic Agar Media and the Rosco Rapid Carb Screen Kit for Detection and Confirmation of Carbapenemase Production in Gram-Negative Bacilli. Journal of Clinical Microbiology; 53(1): 105-112.

10. Chen L, Mediavalla JR, Endimiani A et al. (2011): Multiplex real-time PCR assay for detection and classification of Klebsiellapneumoniae carbapenemases gene $\left(b l a_{K P C}\right)$ variants. Journal of Clinical Microbiology; 49: 579-585.

11. Sekyere JO, Govinden U and Essack SY (2015): Review of established and innovative detection methods for carbapenemase-producing Gram-negative bacteria. Journal of Applied Microbiology; 119: 1219-1233.

12. Nordmann P, Poirel L and Dortet L (2012): Rapid detection of carbapenemase-producing Enterobacteriaceae. Emerging Infectious Disease; 18(9): 1503-1507. 
13. Dortet L, Poirel L, Errera C and Nordmann P (2014): CarbAcineto NP Test for Rapid Detection of Carbapenemase- Producing Acinetobacter spp. Journal of Clinical Microbiology; 52 (7): 2359-2364.

14. Panagea T, Galani I, Souli M, et al., (2011): Evaluation of CHROMagar KPC for the detection of Carbapenemaseproducing Enterobacteriaecein Rectal Surveillance Culture. International journal of antimicrobial agents; 37(2): 124-128.

15. Monterio J, Widen R, Pignatari A, et al., (2012): Rapid detection of carbapenemase genes by multiplex real-time PCR. Journal of Antimicrobial Chemotherapy; 67(4): 906-909.

16. Vasoo S, Cunningham SA, Peggy C Kohner PC, et al., (2013): Comparison of a Novel, Rapid Chromogenic Biochemical Assay, the Carba NP Test, with the Modified Hodge Test for Detection of Carbapenemase-Producing Gram-Negative Bacilli. Journal of Clinical Microbiology; 51(9): 3097-3101.

17. Tijet N, Boyd D, Patel SN, et al., (2013): Evaluation of the Carba NP Test for Rapid Detection of Carbapenemase-Producing Enterobacteriaceae and Pseudomonas aeruginosa. Antimicrobial Agents and Chemotherapy; 57(9): 4578-4580.

18. Yusuf E, Van Der Meeren S, Schallier A, et al.,(2014): Comparison of the Carba NP test with the Rapid CARB Screen Kit for the detection of carbapenemase-producing Enterobacteriaceae and Pseudomonas aeruginosa. European Journal of Clinical Microbiology and Infectious Diseases; 33: 2237-2240.

19. Knox J, Jadhav S, Sevior D, et al., (2014): Phenotypic Detection of Carbapenemase Producing Enterobacteriaceae by Use of Matrix-Assisted Laser Desorption Ionization Time of Flight Mass Spectrometry and the Carba NP Test.
Journal of Clinical Microbiology; 52(11): 4075-4077.

20. Osterblad M, Hakanen AJ and Jalava J (2014): Evaluation of the Carba NP test for carbapenemase detection. Antimicrobial Agents and Chemotherapy; 58: 7553-7556.

21. AbdelGhani S, Thomson GK, Snyder JW, et al., (2015): Comparison of the Carba NP, modified Carba NP, and updated Rosco Neo-Rapid Carb kit tests for carbapenemase detection. Journal of Clinical Microbiology; 53: 3539-3542.

22. Srisrattakarn A, Lulitanond A, Wilailuckana C, et al., (2016): Modification and evaluation of the Carba NP test by use of paper strip for simple and rapid detection of carbapenemase-producing Enterobacteriaceae. World Journal of Microbiology and Biotechnology; 32(7): 117.

23. Dortet L, Poirel L and Nordmann $P$ (2012): Rapid Detection of Carbapenemase-Producing Pseudomonas spp. Journal of Clinical Microbiology; 50(11): 3773-3776.

24. Huang T, Berhin C, Bogaerts P, et al., (2014): Comparative Evaluation of Two Chromogenic Tests for Rapid Detection of Carbapenemase in Enterobacteriaceaeand in Pseudomonas aeruginosa Isolates. Journal of Clinical Microbiology; 52(8): 3060-3063.

25. Heinrichs A, Huang TD, Berhin C, et al., (2015): Evaluation of several phenotypic methods for the detection of carbapenemase-producing Pseudomonas aeruginosa. European Journal of Clinical Microbiology and Infectious Diseases; 34(7): 1467-1474.

26. Adler A, Navon-Venezia S, Moran-Gilad J, et al., (2011): Laboratory and clinical evaluation of screening agar plates for detection of carbapenem-resistant Enterobacteriaceae from surveillance rectal swabs. Journal of Clinical Microbiology;49:2239-2242. 
27. Samra Z, Bahar J, Madar-Shapiro L, et al. (2008): Evaluation of CHROMagar KPC for Rapid Detection of CarbapenemResistant Enterobacteriaceae. Journal of Clinical Microbiology; 46(9): 3110-3111.

28. Gilad JM, Carmeli V, Schwartz D, NavonVenezia S(2011): Laboratory evaluation of the CHROMagar KPC medium for identification of carbapenem-non susceptible Enterobacteriaceae. Diagnostic microbiology and infectious disease ;70(4):565-567. 